In Cres. Vol. $4 N^{\circ} 1:$ pp. 175-184, 2013

\title{
SOFTWARE LIBRE COMO ALTERNATIVA A LAS BARRERAS QUE IMPIDEN INCORPORAR LA TECNOLOGIA EN LA PEQUEÑA Y MEDIANA EMPRESA*
}

\author{
FREE SOFTWARE AS A ALTERNATIVE TO THE BARRIERS \\ THAT PREVENT TECHNOLOGY TO BE INCORPORATED \\ IN SMALL AND MEDIUM ENTERPRISES
}

Anthony José Gomez Morales ${ }^{1}$

\begin{abstract}
RESUMEN
El costo es la principal barrera que tienen las pequeñas y medianas empresas, para incorporar la tecnología en sus actividades. Estas empresas no pueden permitirse el lujo de hacer grandes inversiones sin estar seguras de su rentabilidad. Por tanto, a diferencia de las grandes empresas, no es posible hacer grandes inversiones en el desarrollo de software a la medida de sus requerimientos. Como alternativa de solución, se presenta el Software Libre, basado en la libertad, amparada y garantizada en el adecuado uso del licenciamiento del software, para este caso las licencias libres. Se han establecido cuatro objetivos en el presente artículo: 1) Introducir al lector en los conceptos claves del software libre; 2) Presentar los obstáculos que tienen las pequeñas y medianas empresas para incorporar mejoras; 3) Reflexionar sobre la relación entre las características del software libre y los requerimientos de flujo de información; 4) Incitar al emprendedor a usar el software libre y comprobar sus ventajas prácticas para sus pequeñas y medianas empresas.

PALABRAS CLAVE: Aplicaciones libres, código fuente, copyright, copyleft, estándares abiertos, GNU GPL, licencia, software libre, pequeñas y medianas empresas, ERP, CRM, BI.

* Recibido: 20 de junio del 2013; aprobado: 30 de junio del 2013.

1 Ingeniero Informático. Postgrado en Dirección de Sistemas de Información-Universidad Oberta de Cataluña-Barcelona España. Maestría en Pedagogía Universitaria (e). Docente Universitario. Docente invitado al Programa Titulación Profesional Extraordinarias para Bachilleres en Educación y Segunda Especialidad en Docencia Universitaria-UPAO.

Teléfono: 51044 (949362161). E-mail: anthonyjose@gmail.com
\end{abstract}




\section{ABSTRACT}

The main barrier of small and medium enterprises, to incorporate technology into their activities is cost. They cannot afford to make large investments without being sure of their profitability. Thus, unlike large companies, it is not possible to invest heavily in the development of software to suit your requirements. As an alternative solution, it is presented to Free Software, based on freedom, protected and guaranteed in the proper use of software licensing, in this case the free licenses. Four objectives have been established in this article: 1) To introduce the reader to the key concepts of free software; 2) To present the obstacles of small and medium enterprises to incorporate improvements; 3) To encourage reflection on the relationship between the characteristics of free software and information flow requirements of small and medium enterprises; 4) Encourage the entrepreneur to use free software and verify its practical advantages for small and medium enterprises.

KEY WORDS: Free applications, source code, copyright, copy left, distribution, open standards, GNU GPL, license, free software, small and medium enterprises, ERP, CRM, BI.

\section{INTRODUCCIÓN}

Uno de los múltiples factores que debe tomar en cuenta un emprendedor, cuando establece su propio negocio, es saber cómo va a gestionar todos los flujos de información que debe atender, para satisfacer los requerimientos de: gestión de clientes, contabilidad, logística, relación con el cliente, etc. El uso intensivo de las Tecnologías de la Información y de la Comunicación (TIC) y de los Sistemas de Información(SI) proporcionan un conjunto de herramientas para atender dichos requerimientos. En este sentido, existe un importante grupo de aplicaciones, cuyas características orientadas a la libertad, y que traen importantes consecuencias, se muestran como una alternativa de solución a las barreras que impiden a las pequeñas y medianas empresas (PyME) incorporar la tecnología en sus actividades de flujo de información.

\section{SOFTWARE LIBRE}

Antes de definir lo que es el Software Libre, debemos mencionar a un personaje importante, fundador de esta filosofía de desarrollo de software, Richard Matthew Stallman, quien es también principal impulsor y reconocido por el establecimiento de un marco de referencia moral, político y legal para el movimiento del Software Libre a través del Proyecto GNU(Gnu 2012) y la Fundación de Software Libre (Fsf 2012).

Mantendré esta definición de Software Libre para mostrar claramente qué debe cumplir un programa de software concreto para que se le considere Software Libre. Según (Stallman 2002): 
Software Libre (Free Software) es un asunto de libertad, no de precio. Para entender el concepto, se debe pensar en "libre" como en "libertad", no como en "gratis" (en inglés el término free además de 'libre' significa 'gratis', lo que genera confusión).

Software Libre se refiere a la libertad de los usuarios para ejecutar, copiar, distribuir, estudiar, cambiar y mejorar el software. De modo más preciso, se refiere a cuatro tipos de libertades de los usuarios del software:

- Libertad 0: la libertad para usar el programa sea cual sea nuestro propósito.

- Libertad 1: la libertad para estudiar el funcionamiento del programa y adaptarlo a las necesidades, el acceso al código fuente es condición indispensable.

- Libertad 2: la libertad para redistribuir copias y ayudar así a otros.

- Libertad 3: la libertad para mejorar el programa y luego publicarlo para el bien de toda la comunidad, el acceso al código fuente es condición para esto.

Software libre es cualquier programa cuyos usuarios gocen de estas libertades. De modo que deberías ser libre de redistribuir copias con o sin modificaciones, de forma gratuita o cobrando por su distribución, a cualquiera y en cualquier lugar. Gozar de esta libertad significa, entre otras cosas, no tener que pedir permiso ni pagar para ello. Asimismo, deberías ser libre para introducir modificaciones y utilizarlas de forma privada, ya sea en tu trabajo o en tu tiempo libre, sin siquiera tener que mencionar su existencia. Si decidieras publicar estos cambios, no deberías estar obligado a notificárselo a ninguna persona ni de ninguna forma en particular.

Las libertades antes mencionadas son posibles de mantenerse con el uso adecuado de las Licencias de Software que utilizan los desarrolladores de Software Libre, como las que acuñan adecuadamente el copyleft. Para decidir si una licencia específica puede calificarse de licencia de Software Libre, debemos determinar si se ajusta al espíritu y a las libertades. La Figura 01, muestra el contexto en cual se ubica el Software Libre.

Copyleft es la forma general de hacer un programa Software Libre y requiere que todas las modificaciones y versiones extendidas del programa sean también Software Libre. Utilizaré la traducción de la definición de la Free Software Fundation (Fsf. 2012): 


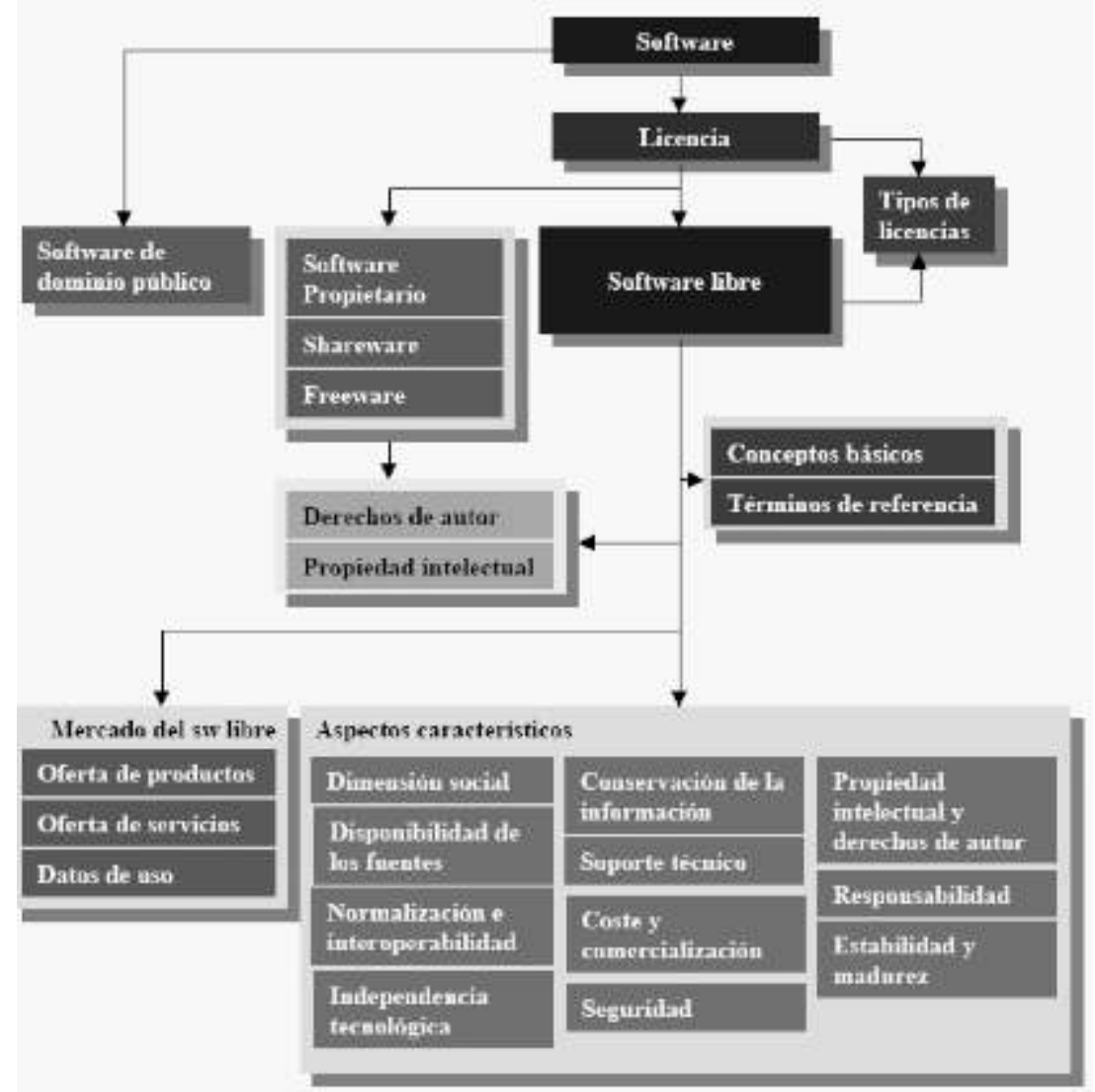

Figura 1. Contexto del Software Libre Fuente: (Curso Software Libre, 2004).

El copyleft usa la ley de copyright, pero le da vuelta para servir a lo opuesto de su propósito usual: en lugar de ser un medio de privatizar el software, se transforma en un medio de mantener libre al software.

La implementación específica de copyleft que se usa para la mayoría del software del Proyecto GNU es la Licencia Pública General de GNU (GNU General Public License) o GNU GPL.

\section{LAS PYMES, TECNOLOGÍA DE LA INFORMACIÓN (TI) Y LA SOCIEDAD DE LA INFORMACIÓN}

La legislación peruana define a la Micro y Pequeña Empresa (MYPE), según Ley 28015 Ley de Promoción y Formalización de la Micro y Pequeña 
Empresa, como: “...la unidad económica constituida por una persona natural o jurídica, bajo cualquier forma de organización o gestión empresarial contemplada en la legislación vigente, que tiene como objeto desarrollar actividades de extracción, transformación, producción, comercialización de bienes o prestación de servicios (...) debiendo contar con las siguientes características:

- Microempresa: Número total de trabajadores entre uno (01) y diez (10). Niveles de ventas anuales no mayores a 150 UIT.

- Pequeña empresa: Número total de trabajadores hasta un máximo de cien (100). Niveles de ventas anuales hasta un máximo de 1700 UIT.

Para los efectos del presente documento, mantendremos la acepción de Pequeñas y Medianas empresas (PyME).

Las Tecnologías de la Información y las Comunicaciones (TIC), son el conjunto de herramientas, equipos, programas informáticos, aplicaciones, redes y medios, que permiten la compilación, procesamiento, almacenamiento, transmisión de información como: voz, datos, texto, video e imágenes. Las TIC constituyen el acontecimiento cultural y tecnológico de mayor alcance y expansión del último siglo y lo transcurrido del presente. Nuestro país no escapa a tal proceso de globalización de la sociedad de la información, y las PyME no son la excepción, aunque con indicadores no muy alentadores, de acuerdo a los resultados de la Encuesta de Micro y Pequeña Empresa (EMYPE 2010):

- Durante el año 2009, el 5,5\% de los conductores de las MYPE participaron en eventos de servicios sobre Nuevas Tecnologías para la Gestión. Las ciudades que registran los mayores porcentajes de capacitación en este tipo de tecnologías son Chiclayo con $9,8 \%$; Iquitos, con $8,1 \%$; y Huancayo, con $7,3 \%$; en tanto que, los porcentajes más bajos se registran en Arequipa con 6,9\%; Piura, 6,6\%; Lima-Callao 5,2\% y Trujillo con 4,6\%.

- Durante el año 2009, el 5,6\% de las micro y pequeñas empresas participaron en cursos y/o servicios de capacitación sobre TIC. Lima-Callao, Chiclayo y Piura, registraron los mayores porcentajes con $6,1 \%, 5,8 \%$ y $4,4 \%$, respectivamente, en tanto que las ciudades con menores porcentajes son: Huancayo e Iquitos con 3,6\% cada uno, Arequipa y Trujillo con $3,4 \%$ y $1,1 \%$, respectivamente.

- Durante el periodo de referencia, el porcentaje de empresas que optaron por capacitarse en tecnologías para mejorar sus procesos, produc- 
tos o servicios fue del 11,4\%. Los valores más altos del indicador se muestran en: Piura con 15,4\%, seguido de Lima-Callao, con 11,8\%, Arequipa $10,2 \%$, Huancayo $9,7 \%$ y Chiclayo con $9,4 \%$, en tanto que los menores porcentajes se registran en Iquitos y Trujillo con $6,6 \%$ y $6,3 \%$, respectivamente (Tabla 01 ).

Tabla 01

MYPE QUE ADOPTAN TECNOLOGÍAS PARA MEJORAR PROCESOS PRODUCTIVOS, SEGÚN CAPITAL DEPARTAMENTAL, 2009

\begin{tabular}{lccc}
\hline $\begin{array}{c}\text { Capital } \\
\text { departamental }\end{array}$ & MYPE & $\begin{array}{c}\text { MYPE que participaron en proyectos y/o } \\
\text { eventos de capacitación para la aplicación o } \\
\text { introducción de nuevas o mejores } \\
\text { tecnologías innovadoras para mejorar } \\
\text { productos, procesos o prestación } \\
\text { de servicios }\end{array}$ & $\begin{array}{c}\text { Indicador } \\
(\%)\end{array}$ \\
\hline Total & 12544 & 1427 & 11,4 \\
Lima - Callao & 10416 & 1233 & 11,8 \\
Arequipa & 655 & 67 & 10,2 \\
Trujillo & 571 & 36 & 6,3 \\
Chiclayo & 276 & 26 & 9,4 \\
Iquitos & 197 & 13 & 6,6 \\
Huancayo & 247 & 24 & 9,7 \\
Piura & 182 & 28 & 15,4 \\
\hline
\end{tabular}

Fuente: INEI-Encuesta de Micro y Pequeña Empresa.

\section{BARRERAS QUE IMPIDEN INCORPORAR LAS TI EN LAS PYMES}

Actualmente la tecnología ha resultado ser vital para las PyME, debe ser una herramienta integrada en los procesos de estas organizaciones. Es un catalizador de innovación y transformación en las empresas. Las PyME necesitan incorporar la tecnología a sus estrategias de negocio para poder ser más productivas y aumentar su grado de eficiencia; pero existen diversos obstáculos que impiden el desarrollo de proyectos de tecnología en las PyME. Existen cuatro barreras principales que impiden que las PyME logren su propio cambio, innovación y modernización (Valenzuela, 2003): la resistencia al cambio; la definición de requerimientos; el hardware y el software; y la dependencia de los proveedores de tecnología, específicamente del software.

La resistencia al cambio incluye diferentes aspectos como el temor al uso 
de tecnología por parte de los trabajadores; los errores en el uso de la nueva tecnología; el cambio de cultura y comportamiento; y la escasa participación de los usuarios finales en el levantamiento de los requerimientos, el diseño y el desarrollo de las aplicaciones.

La segunda barrera se refiere a la incorrecta definición de los requerimientos de la aplicación que se quiere instalar, sea específica o estándar.

Por otro lado está el obstáculo del hardware y el software, pues muchas de las empresas no cuentan con un soporte técnico eficiente y los equipos de computadoras existentes son insuficientes para el desempeño de las actividades básicas. En este punto podíamos añadir una barrera adicional: el costo.

En las barreras de costos, las PyME no pueden permitirse el lujo de hacer grandes inversiones sin estar seguras de su rentabilidad. Por tanto, a diferencia de las grandes empresas, no es posible hacer grandes inversiones en el desarrollo de Software a la medida de sus requerimientos y, más aun, con el grado de dependencia que se suele generar con el desarrollador o productor del software. Esta barrera de costos conlleva a que las PyME, para el caso de aplicaciones de software genéricas, realicen actividades ilegales como la piratería de software, práctica que es común pero no menos grave.

\section{SOFTWARE LIBRE Y LAS PYME}

Dos de los dilemas que, en la actualidad, deben enfrentar las PyME, para vencer su principal obstáculo, está relacionado con la exigencia de que este tipo de organizaciones desistan del uso de la piratería como alternativa para satisfacer sus necesidades, de flujos de información, a nivel de software y en cambio, opten por la utilización de aplicaciones licenciadas. No obstante, la mayoría de soluciones con licenciamiento suelen ser costosas, siendo este el segundo dilema y, por lo general, no están al alcance económico de este tipo de negocios.

Así surge la pregunta: ¿Cómo satisfacer los requerimientos de software de las PyME, sin caer en una mala práctica empresarial y arriesgar la gestión de la empresa no incluyéndolas para los requerimientos de flujo de información?

Existe una gran oportunidad para que las PyME logren cubrir sus requisitos de software sin violar permisos y lo mejor, en la mayoría de los casos, a bajo o ningún costo. Esta alternativa se llama Software Libre, un universo de programas útiles, fiables, seguros, estables, con soporte, disponibles de manera libre y la mayoría de manera gratuita para todo tipo de necesidades. No obstante, más allá del precio, hay un aspecto muy importante que permite y defiende la naturaleza de uso de este tipo de herramientas libres: La licencia. 
La licencia de este tipo de aplicaciones de software está sujeta básicamente a cuatro tipos de libertades, que hemos revisado anteriormente, y que benefician directamente a los usuarios, en este caso en particular a las PyME: la libertad para usar el programa para cualquier propósito, la libertad para estudiar el funcionamiento del programa y adaptarlo a tus necesidades, la libertad para redistribuir copias, la libertad para mejorar el programa.

Las aplicaciones de software libre han venido en constante evolución y hoy se consolidan como la solución tecnológica para las PyME que no poseen los recursos económicos suficientes para acceder a las soluciones que el mercado de software privativo tiene para ofrecerles.

Ahora bien, es importante aclarar, de antemano, que la adopción de este tipo de tecnologías, en las PyME, demandará un proceso de adaptación a las necesidades y requisitos de la empresa, así como también, de un proceso de capacitación y entrenamiento sobre el uso del software; que de cierta forma requerirá de alguna inversión por parte de la empresa, pero que, sin duda, estará lejos de lo que podría representar la adquisición de algún servicio de software privativo o propietario.

Una apuesta clara por el software libre para PyME, la constituye OpenPYME, un catálogo de Software Libre donde se recopilan, de forma categorizada, productos sólidos y fiables que pueden incorporarse en cualquier ámbito productivo de una empresa, mejorando así su gestión y competitividad gracias a la inclusión de herramientas TIC, sin tener que pagar por ello. Esta iniciativa de la Universidad de la Laguna-España ofrece diversas aplicaciones libres que van desde software para gestión financiera, hasta aplicaciones de ofimática, pasando por recursos empresariales como CRM (Relaciones con el Cliente), ERP (Planificación de Recursos Empresariales), BI (Inteligencia de Negocios), E-Commerce (comercio electrónico), Gestión de proyectos, entre otros.

\section{APLICACIONES PARA LAS PYMES BASADAS EN SOFTWARE LIBRE}

OpenBravo ERP. Es una aplicación de gestión empresarial del tipo ERP destinada a empresas de pequeño y mediano tamaño. La estructura de datos de la aplicación está basada originalmente en una versión antigua de Compiere. La cobertura funcional del producto incluye todas las áreas típicas de un sistema de gestión integrado (www.openbravo.com/).

Pentaho. Es una plataforma de BI “orientada a la solución” y “centrada en 
procesos". Pentaho consiste en una Suite Completa de Inteligencia de Negocio que incluye todos los principales componentes requeridos para implementar soluciones de BI, tales como: Reporting, Análisis, Dashboard, Data Mining e Integración de datos (http://www.pentaho.com/).

CiviCRM. Es un gestor de relaciones con el cliente (CRM). Está diseñado para satisfacer las necesidades de organizaciones no gubernamentales (ONG), asociaciones sin ánimo de lucro, etc. CiviCRM puede desplegarse independientemente o integrado con gestores de contenido como Joomla o Drupal (http://sourceforge.net/projects/civicrm/files/).

OpenCar. Es una solución de comercio electrónico basada en osCommerce pero bastante más sencilla y reducida. Es un sistema completo de tienda virtual gratuito y de código abierto realizado con PHP y MySQL, que permite montar fácilmente un negocio en internet (http:// www.opencart.com/).

OpenPYME. Es un proyecto promovido por la Oficina de Software Libre de la Universidad de La Laguna - España, bajo el marco de colaboración científicotecnológico entre la Agencia Canaria de Investigación, Innovación y Sociedad de la Información del Gobierno de Canarias y la Universidad de La Laguna. OpenPYME es un catálogo de Software Libre donde se recopilan, de forma categorizada, productos sólidos y fiables que pueden incorporarse en cualquier ámbito productivo de una empresa, mejorando así su gestión y competitividad gracias a la inclusión de herramientas TIC (http://openpyme.osl.ull.es/).

\section{CONCLUSIONES}

El Software Libre representa una oportunidad sin precedentes para las PyME, que afrontan una serie de barreras para incorporar las TIC en su modelo de negocio, principalmente las barreras económicas y de licenciamiento; existente para los países en vía de desarrollo, como el nuestro.

Aprovechar esta oportunidad depende, en gran medida, de las mismas PyME que asuman el reto de asimilar esta alternativa tecnológica para estar dentro de la legalidad y satisfacer sus requerimientos de flujo de información, con el uso de aplicaciones de software, utilizando software libre.

En los últimos años, el Software Libre ha ampliado sus horizontes y ahora se presenta como una mejora para los flujos de información en las PyME, que está al alcance de todas, con programas muy simples que proporcionan excelente calidad y satisfacen las necesidades de los empresas, basado en las consecuencias de la libertad. 


\section{BIBLIOGRAFÍA CONSULTADA}

Ley 28015 (2008). Ley de promoción y formalización de la micro y pequeña empresa, y su modificatoria en el Decreto Legislativo $\mathrm{N}^{\circ} 1086$.Publicado el 28 de junio de 2008.

ENAHO (2011). Encuesta Nacional de Hogares - INEI , http://www.inei.gob.pe/web/Boletin/Attach/ 13382.pdf

UNIVERSIDAD ESAN. (2010). Modelo tecnológico de integración de servicios para la MYPE peruana, Esan, Lima-Peru.

VAlenzuela, JuAn Alonso. (2003). Las tecnologías de información en las PyMEs. www.gestiopolis.com/recursos/documentos/fulldocs/ger1/tipyme.htm

MATterlart, ARMAND. (2003). Historia de la sociedad de la información, Ediciones Paidós. España.

STALLMAN, RichARD. (2002). Free software, free society GNU Press. España.

STALLMAN, RICHARD. (2003). Some confusing or loaded words and phrases that are worth avoiding, http://www.gnu.org/philosophy/words-to-avoid.html.

FREE SOFTWARE FUNDATION. http://www.fsf.org. USA.

PRoYeCto GNU. Gnu no es Unix. http://www.gnu.org. USA.

CURSO SOFTWARE LIBRE (2004) de los Doctorados en Informática y Modelización Matemática de la Universidad Rey Juan Carlos y Doctorado en Ingeniería Telemática de la Universidad Politécnica de Madrid. http://gsyc.escet.urjc.es/docencia/asignaturas/doct-sobre/. España. 